\title{
Location Location Location: Experiences of Authoring an Interactive Location-based Narrative
}

\author{
David E. Millard and Charlie Hargood \\ Electronics and Computer Science \\ University of Southampton \\ Southampton, UK SO17 1BJ \\ \{dem, cah07r\}@ecs.soton.ac.uk
}

\begin{abstract}
Location-based narratives are a form of digital interactive storytelling where a reader's physical movement triggers narrative events. Unlike traditional hypertext the poetics of these narratives is poorly understood, meaning that it is difficult to build effective authoring tools or to train new writers. In this paper we present our experience of authoring an interactive location-based narratives, focused on the creation of a story 'The Isle of Brine' set on the island of Tiree. Our experience highlights the primacy of location in the authoring process, and both pragmatic and aesthetic considerations for the design of the narrative.
\end{abstract}

Keywords: Location-based Narratives, Sculptural Hypertext

\section{Introduction}

The popularity of smart-devices with location awareness has led to increasing interest in location-based interactive narratives. These are digital stories, read on a smart-device, which react or change to the reader's location $[1,5,7]$. However, little work has been undertaken to understand the poetics of location-based writing (in contrast to the body of theory on hypertext writing and poetics $[6,9])$. Without this understanding it is difficult to produce effective tools for creating location-based narratives, or to educate writers about the possibilities.

The StoryPlaces project is a collaboration between Computer Scientists and English Scholars to explore the poetics of location-based narratives in order to inform the creation of authoring tools and support writers.

There are models of hypertext that can be applied. Sculptural Hypertext sees all nodes as potentially available, but sculpts away certain nodes based on the user's state, and conditions and rules on the nodes. If one makes user location a variable that can be used for conditions, and changed by rules, then the model becomes sufficient to describe most location-based narratives [4].

In StoryPlaces we are exploring the issues around authoring interactive locationbased stories based on sculptural hypertext models. However, as technologists it became clear that our understanding would always be deficient unless we attempted to create a story ourselves. The Tiree Tech Wave provided us with the 
opportunity of a safe space [8] where we could take on the role of authors and the risk of failure was minimal. We attended the April 2016 Tech Wave, and spent five days on the island, using this time to research local history, explore locations, and draft a story structure in the format required for our technology.

\section{Methodology and Experience}

Experiential Inquiry is a qualitative approach where "the agent himself (sic) engages systematically in a self-directed exploration of his own experience and behaviour and attends fully to the experience and behaviour of other agents who are similarly engaged in interaction with him" [3]. For us the creation of a location-based story on the island was the focus of the inquiry, and the Tech Wave the mechanism by which we came together to engage with the task.

We began on day one by discussing key themes, the starting premise, and the narrative and sculptural structures that we might use. We wanted our story to reflect our own experience, and so decided that our protagonist should be a visiting professional in the spirit of Lovecraft's 'The Mountains of Madness'. The conceit being that it is the expert whose view is changed by the experience.

We decided on a basic structure for the story, based around three acts unfolding across three separate areas of the island. This would enable readers to drive between acts, but then walk the nodes of the story itself. It was clear that there were excellent local resources about the history and myths of the island, and our idea was to link these with our protagonist as the reader progressed through the Acts of the story. a website that mapped English, Gaelic and Norse place names to survey data from around the island. This inspired us to make our protagonist a surveyor, and we reasoned we could use this resource to populate a number of nodes about the island and its history. Act 1 would be played straight, with our surveyor exploring the island and using this historical information. Act 2 would begin to hint at some connection to the island, and begin to blur fact and fiction. While Act 3 would disolve into delusion and reveal some connection between the surveyor and the island and its legends.

With the help of local expertise we drafted a list of local stories, identified three key areas of the island, and created a simple written manifest of locations for each area, listing the sorts of location we would need to match our stories. On the following two days we visited the areas, located appropriate starting places (where we could park), and then walked around the area using a GPS tool to record location stamps. Our choice of location stamps was partly practical (was it on an effective path or route), partly logical (could you see things that were referenced in the stories), and partly aesthetic (was the location evocative of the things described in the stories).

\section{Our Story: The Isle of Brine}

As part of our project we had already undertaken some analysis work around patterns of sculptural hypertext [2]), and decided that we would use a version of 
the Phasing pattern to manage the high level structure of our story. Phasing is where a story progresses through a number of distinct phases, typically nodes are available in any order within a given phase, with particular nodes transitioning the whole system to the next phase. A typical example would be to support a three-act structure, where each act is a separate phase.

Isle of Brine uses two three act structures that develop independently. This is similar to another pattern, parallel threads, but with two entire phases progressing in parallel rather than two sequences of nodes. One (the Island phases) moves forward in time from the surveyors arrival on the island, the other (the History phases) moves backwards. The Island phases develop the surveyors experiences on the Island and contain revelations about his past. The History phase develops the story of how he came to travel to the island, and contains revelations about his motivations. Readers experience of the story thus varies according to the juxtaposition of phases, and the order in which the revelations are made.

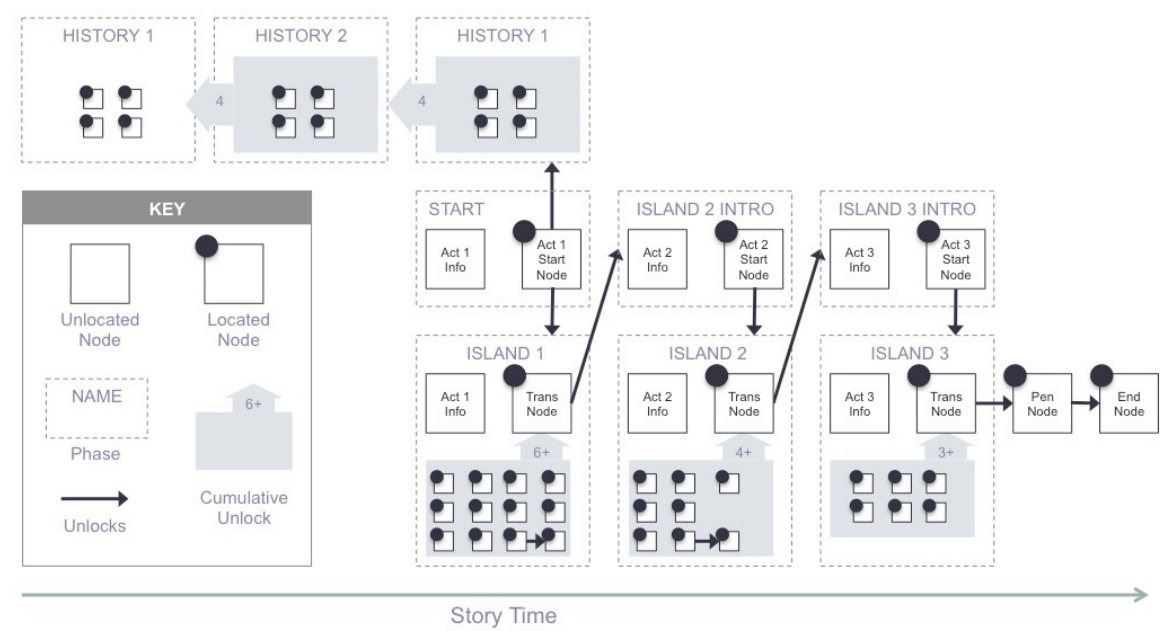

Fig. 1. The sculptural structure of 'Isle of Brine'

The main phases are the ones for the Island. In each act there is an intro phase, with a start node at a specific location, once this has been read it moves into the main phase that contains the remaining nodes. In both phases an unlocated node (which is always available regardless of location) gives some context for the current state of the story. Specific transition nodes exist in the main phases that move the acts along, but we use another pattern Unlocking to ensure that a certain number of nodes are read in each phase before the transition node becomes available. The secondary phases are the ones for History. In these cases all the nodes in the phase must be read before the phase changes to the next one. Each node in the history phases is mapped to three alternative locations, one for each area used by the Island nodes. Thus as long as the appropriate History 
phase is active, they can be read in a location that is near to wherever the current act is playing out. Figure 1 shows a graphical version of the structure.

\section{Conclusions}

In this paper we have described how a journey to the Tiree Tech Wave created a safe space in which we could explore the authoring process ourselves, pushing the technology beyond what was being done in the main project activities, and confronting directly the issues that we had previously only seen second hand. The results is a story 'The Isle of Brine' that goes beyond the typical hypertextual complexity of a location-based story ${ }^{1}$. Despite our focus on the non-linear structure our experience has highlighted the primacy of location in the authoring process, and revealed a number of ways that this has a pragmatic and aesthetic impact on the narrative. Rather than a neatly layered process, where stories can be laid down upon locations, we have discovered a deeply interconnected set of decisions that show that narrative structure, location topology, and the events of the story itself are co-dependent. Our view is that authors must design, select and develop these simultaneously, or at least iteratively, in order to create successful location-based stories.

\section{References}

1. J. Broadbent and P. Marti. Location aware mobile interactive guides: usability issues. In Proceedings of the Fourth International Conference on Hypermedia and Interactivity in Museums (ICHIM97), pages 162-172, 1997.

2. C. Hargood, V. Hunt, M. Weal, and D. E. Millard. Patterns of sculptural hypertext in location based narratives. In Proceedings of the 27th ACM Conference on Hypertext and Social Media, HT '16, New York, NY, USA, 2016. ACM.

3. J. Heron. Experience and method. an inquiry into the concept of experiential research. Technical report, University of Surrey, UK, 041971.

4. D. E. Millard, C. Hargood, M. O. Jewell, and M. J. Weal. Canyons, deltas and plains: Towards a unified sculptural model of location-based hypertext. In Proceedings of ACM Hypertext 2013, pages 109-118, New York, USA, 2013. ACM.

5. V. Nisi, I. Oakley, and M. Haahr. Location-aware multimedia stories: turning spaces into places. Universidade Cátolica Portuguesa, pages 72-93, 2008.

6. M. Pisarski. New plots for hypertext?: Towards poetics of a hypertext node. In Proceedings of Hypertext 2011, pages 313-318, New York, NY, USA, 2011. ACM.

7. F. Pittarello. Designing a context-aware architecture for emotionally engaging mobile storytelling. INTERACT 2011, pages 144-151, 2011.

8. P. Reason. Editorial Introduction: The Practice of Co-operative Inquiry. Systemic Practice and Action Research, 15(3):169-176, 2002.

9. J. Walker. Piecing together and tearing apart: Finding the story in afternoon. In Proceedings of ACM Hypertext '99, pages 111-117, New York, USA, 1999. ACM.

\footnotetext{
${ }^{1}$ We would like to thank Professor Alan Dix for his support during the Tiree Tech Wave, our fellow participants who contributed enormously to our experience, and our colleagues on StoryPlaces - funded by The Leverhulme Trust (RPG-2014-388).
} 